age faster than does male fertility, that is, if the ratio $f(t) / m(t)$ increases monotonically with age; protogyny is evolutionarily stable if this ratio decreases ${ }^{6,17,18}$. Female fertility may often be determined by fecundity and should increase with age. Male fertility may also usually increase with age, but the extent of this increase will depend on the social system. The greatest potential for an increase in $m(t)$ with $t$ should be associated with extreme polygyny, hence we expect that protogyny will be associated with polygyny. Protandry, on the other hand, should be found in species in which single males do not monopolize many matings.

If fertility increases with age for both sexes, a protandrous male should be most fit just before changing sex whereas a protandrous female is least fit just after changing sex, so

$$
\frac{\bar{M}}{m(\tau)}<1<\frac{\bar{F}}{f(\tau)}
$$

From results 4 and 5 we expect a male excess under protandry, and a male excess is nearly always observed (Table 1). Under protogyny, these inequalities should be reversed, and the expected female excess is also supported by the data. The obvious alternative hypothesis - that the second sex is rarer because the brief period of sexual transition involves high mortality-finds no support in observations on sex change in fish ${ }^{17-19}$.

Several of the protogynous fish listed in Table 1 exhibit social systems in which a few males dominate a social group and appear to prevent females from changing sex. Removal of a male leads to rapid sex change of the largest or most dominant female ${ }^{19}$. Such systems are easily incorporated into the foregoing framework, except that fertility is a discrete rather than continuous function of group size. Equality 3 then becomes an inequality, and the sex-ratio skew will be even greater than predicted by equation 4 . Anemonefish (whose observed sex ratio is 0.5 ) provide a special case because the social system limits group size to two individuals, in which case male fertility depends entirely on group composition, not age. The limpet Crepidula convexa also violates the usual protandry sex-ratio pattern; the reason for this exception is unexplained but may stem from the mobility of males, which may offer an advantage to small size $[m(t)$ would decrease with $t]$.

Sex ratios under environmental sex determination are also skewed from 0.5 , favouring males in a mermithid nematode ${ }^{22}$ and females in reptiles (data were summarized in ref. 8). A mermithid's sex is determined in response to nutrition during its juvenile phase when it parasitizes an insect: a worm developing in an undernourished, crowded, and or/small host becomes a male, whereas a worm developing in a large, well-nourished host becomes a female ${ }^{6,22}$. The mermithid case thus parallels protandry. The primary sex ratio lies in the range 0.7-0.9 for each of five populations of the mosquito parasite Romanomermis nielseni, which is remarkable in view of the fact that the distributions of $t$ differ greatly among populations ${ }^{22}$.

Data from a variety of turtles and crocodilians with temperature-dependent sex determination suggest that primary sex ratios are often as extreme as 0.3 , although the sex ratio in some species is consistent with 0.5 (ref. 8). These skewed sex ratios fall within the range observed in sex-changing fish and mermithids, but it is not yet plausible that incubation temperature has such large effects on fertility ${ }^{21}$.

The present theory offers the best explanation for overall trends in the data in that the direction of observed sex-ratio excesses are predicted (except in Crepidula). The observed sexratio excesses are often extreme, particularly under protogyny and in the nematodes. The present theory can potentially account for the observed magnitudes of deviations from 0.5 , but quantitative predictions await studies of the actual fertility functions, $m(t)$ and $f(t)$. Perhaps the most interesting aspect of this theory as applied to sex change is that the generalizations apply to the adult or breeding sex ratio. Under typical fisherian sex-ratio selection for dioecious species, it is the primary sex ratio that is predicted to obey a generalization (equal investment), and the adult sex ratio is simply a consequence of the prevailing sex-specific mortality rates.
Received 21 November 1988; accepted 31 January 1989

1. Fisher, R. A. The Genetical Theory of Natural Selection (Clarendon, Oxford, 1930)

2. Maynard Smith, J. Evolution and the Theory of Games (Cambridge Univ. Press, Cambridge, 1982).

3. Shaw, R. F. \& Mohler, J. D. Am. Nat. 87, 337-342 (1953).

4. Williams, G. C. Adaptation and Natural Selection (Princeton Univ. Press, Princeton, 1966).

5. Leigh, E. G. Oxford Surveys evol. Biol. 3, 187-223 (1986).

6. Charnov, E. L. The Theory of Sex Allocation (Princeton Univ. Press, Princeton, 1982)

7. Hamilton, W. D. Science 156, 477-488 (1967)

8. Bull, J. J. \& Charnov, E. L. Oxford Surveys evol. Biol. 5, 96-135 (1988)

9. Lewis. D. New Phytologist 40,56-63 (1941).

10. Howard, H. W. J. Genet. 44, 143-159 (1942).

11. Hamilton, W. D. A. Rev. Ecol. Syst. 3, 193-232 (1972)

12. Trivers, R. L. \& Hare, H. H. Science 191, 249-263 (1976).

13. Karlin, S. \& Lessard, S. Theoretical Studies on Sex Ratio Evolution (Princeton Univ. Press, Princeton, 1986).

14. Frank, S. A. \& Swingland, I. S. J. theor. Biol. 135, $415-418$ (1988)

15. Charnov, E. L. \& Bull, J. J. J. theor. Biol. (in the press).

16. Bull, J. J. Heredity 46, 9-26 (1981)

17. Warner, R. R. Environmental Biology of Fishes Vol. 22, 81-90 (1988)

18. Warner, R. R. Trends Ecol. Evol. 3, 133-136 (1988)

19. Shapiro, D. Y. Science 209, 1136-1137 (1980).

20. Trivers, R. L. \& Willard, D. E. Science 179, 90-92 (1973)

21. Bull, J. J. Evolution of Sex Determining Mechanisms (Benjamin/Cummings, Mento Park, 1983)

2. Blackmore, M. S. thesis, Univ. Utah (1989).

23. Charnov, E. L. Am. Nat. 113, 715-734 (1979)

24. Charnov, E. L., Gotshall, D. \& Robinson, J. Science 200, 204-206 (1978)

25. Ballantine, W. J. thesis, London Univ. (1961)

26. Hoagland, K. E. Malacologia 17, 365-391 (1978)

27. Robertson, D. R. \& Warner, R. R. Smithsonian Contrib. Zool. 255, 1-26 (1978)

8. Choat, J. H. \& Robertson, D. R. in Intersexuality in the Animal Kingdom (ed. Reinboth. R.) 263-283 (Springer, Berlin, 1975).

29. Warner. R. R. \& Robertson, D. R. Smithsonian Contrib. Zool. 254, 1-27 (1978)

30. Robertson, D. R. \& Choat, J. H. Proc. Second Int Symp. Coral Reefs I (Biology), 217-225 (1974)

31. Moe, M. A. Florida Dept. Nat. Resources Prof. Paper 10, 1-95 (1969).

32. Shapiro, D. Y. \& Lubbock, R. J. theor. Biol. 82, 411-426 (1980).

33. Fricke, H. \& Fricke, S. Nature 266, 830-832 (1977).

34. Fricke, H. W. Z Tierpsychol, 50, 313-326 (1979).

35. Moyer, J. T. \& Nakazono, A. Jap. J. Ich. 25, 25-39 (1978)

\title{
Production of chimaeric mice containing embryonic stem (ES) cells carrying a homoeobox Hox 1.1 allele mutated by homologous recombination
}

\section{Andreas Zimmer \& Peter Gruss}

Max-Planck Institut für biophysikalische Chemie

Abt. Molekulare Zellbiologie, Am Fassberg, 3400 Göttingen, FRG

SEVERAL mouse gene families related to Drosophila developmental control genes and containing a homoeobox, a paired box or a finger domain, have been cloned and structurally analysed. On the basis of structural similarities to the Drosophila genes and of their spatially and temporally restricted expression patterns during mouse embryogenesis, it has been proposed that these mammalian genes also are involved in the control of development $^{1-4}$. To elucidate the function of homoeobox genes by genetic means, mouse mutants must be generated. We have developed a technique for mutagenesis in vivo and have used it to mutate the homoeobox Hox 1.1 gene. In vivo mutagenesis was achieved through homologous recombination between an endogenous $H o x$ 1.1 allele and a microinjected mutated gene in pluripotent embryonic stem (ES) cells ${ }^{5-9}$. Mutant cells were identified by means of the polymerase chain reaction (PCR) ${ }^{10}$ and mutant clones were used to generate chimaeric mice. Because the homologous recombination event is formally a gene conversion event and no selection is required to screen for cells carrying the mutated allele, in vivo mutagenesis allows specific alterations in the target sequence to be made without the introduction of any other sequences. 
FIG. 1 Strategy for the mutagenesis of the Hox 1.1 gene and its detection by PCR. An Fspl fragment of the Hox 1.1 gene (nucleotides 367-1937 relative to the translational start codon, see Fig. 3) was cloned into Bluescript (Stratagene) and a 20-bp oligonucleotide inserted into the EcoRI site of the homoeobox. Homologous recombination of this fragment with the endogeneous Hox 1.1 gene in the recipient cells introduced this oligonucleotide into the endogenous gene. Consequently, transcripts from this allele had a frameshift mutation with a stop codon directly downstream of the oligonucleotide in-frame. To screen the recipient cells for homologous recombination events by PCR, we synthesized two primers, of which the first (Primer 1; TTC CGC ATC TCA CCC TGG AT) was specific for the first exon of Hox 1.1, binding $5^{\prime}$ to the Fspl site, and the second (Primer 2; AAT TGT GAG GTA CCG CTG AC) was identical to the inserted oligonucleotide. As the normal Hox 1.1 allele contained no binding site for primer 2 and the microinjected fragment contained no binding site for primer 1 , only the mutant allele can contain both priming sites. The homoeobox is indicated by a dark-shaded box. Primer 1 and its binding site are depicted as a light-shaded arrowhead and box, respectively. Primer 2 and the oligonucleotide are shown as a black arrowhead and box, respectively.

Relatively few genes are amenable to screening for homologous recombination using selective conditions ${ }^{11,12}$. We therefore designed a general strategy for targeting specific mutations to genes avoiding the need for selection. We used microinjection of DNA because it is the most efficient method for introducing DNA into cells $s^{13}$ and increases the ratio of homologous to illegitimate recombination (M. R. Capecchi, personal communication). We limited the amount of nonhomologous DNA to the introduced mutation and we used the PCR to screen cell pools for homologous recombination events. We used this procedure to interrupt the homoeobox of a Hox 1.1 allele with a 20 -base pair (bp) oligonucleotide. The protein encoded by the resulting mutated allele has an intact aminoterminus and a truncated carboxy-terminus and consequently lacks the putative DNA binding domain. The oligonucleotide sequence used not only ensured the introduction of a $\mathrm{KpnI}$ site at the expense of the EcoRI site (see Fig. 3d) but also served as a priming site for the amplification of the Hox 1.1 intron by the PCR technique.

To obtain substrate for homologous recombination, the intron and second exon of the Hox 1.1 gene ${ }^{15,16}$ were subcloned and a 20-bp oligonucleotide was inserted into the EcoRI site of the homoeobox (Fig. 1). To test the efficiency of homologous recombination and the specificity of the PCR analysis, the Fsp Ifragment (see Figs 1 and 3 ) was first injected into large pools (250-600 cells) of 3T3 and P19 cells. As outlined in Fig. 1, the PCR with genomic DNAs using primers 1 and 2 (arrowheads in Fig. 1) will amplify a 1.1-kilobase (kb) DNA fragment including the intron-exon boundaries of Hox 1.1 only if the microinjected fragment has correctly recombined with the genomic DNA. Figure $2 a$ shows the amplification of the predicted $1.1-\mathrm{kb}$ band from two of the microinjected pools (lane 1 and 2), compared with the negative signal from a third pool (lane 3 ). The plasmid $p \mathrm{H} 1.1 / \mathrm{O}-\mathrm{BF}$ (Fig. $2 c$ ), which includes the entire Hox 1.1 gene and the inserted oligonucleotide, served as a positive control (lane 4). A total of 3,180 cells in seven pools were microinjected and at least five independent homologous recombination events were detected. The frequency of homologous recombination was therefore higher than in 1 in 640 cells, and individual events could be detected among large cell pools.

Having established the reliability of the PCR analysis of homologous recombination, we injected the FspI fragment into

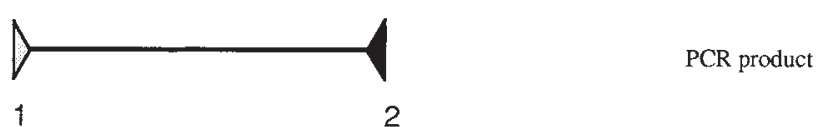

pools of 50-200 ES cells growing in 6-cm tissue culture dishes. The ES cell line D3 (ref. 7) was used because it contributes to the germ line of chimaeric animals and maintains this capability after genetic manipulations ${ }^{9}$. Figure $2 b$ shows the PCR screening of 12 microinjection experiments. Southern blot analysis of the amplified DNA using an intron-specific probe (see Fig. 2c) detected a 1.1-kb fragment in five lanes, corresponding to at least five independent recombination events. Table 1 summarizes the analysis of the microinjected ES cell pools. The average frequency of homologous recombination was 1 event per 150 microinjected cells.

Two ES cell pools ( 1 and 7; Fig. $2 b$ and Table 1 ) were chosen for further analysis. Single cell clones from these pools were obtained by picking individual cells with a glass capillary ${ }^{17}$. DNA was prepared from individual clonal cell lines, digested with $K p n I$ and used for Southern blots. Figure $3 a$ shows the result of one subcloning experiment in which four individual clones were examined. Because the inserted oligonucleotide has a KpnI restriction enzyme site, the appearance of a new 5.4-kb band in homologously recombined clones, in addition to the 14-kb band from the normal allele, was expected after hybridization with a probe specific for the first exon (probe 1 in Fig. $3 d$ ). The presence of this band is shown in lane 1 of Fig. $3 a$. The ES cell DNA became contaminated with feeder cell DNA

TABLE 1 Analysis of microinjected ES cell pools by PCR

\begin{tabular}{ccc}
\hline Pool no. & No. of injected cells & PCR \\
1 & 50 & + \\
2 & 71 & + \\
3 & 54 & + \\
4 & 45 & - \\
5 & 108 & - \\
6 & 130 & - \\
7 & 50 & - \\
8 & 48 & - \\
9 & 42 & - \\
10 & 46 & - \\
11 & 50 & + \\
12 & 25 & \\
\hline
\end{tabular}

Details of the analysis are given in Fig. $3 b$. 
because the ES cells were not allowed to form large colonies during subcloning. Thus, the 14-kb Hox 1.1 fragment represents not only the one wild-type allele from the ES cells, but also both wild-type alleles from the feeder layer cells. In total, 161 individual clones from pool 1 and 165 clones from pool 7 were analysed by Southern blotting; one clone from pool 1 and two clones from pool 7 contained a mutated Hox 1.1 allele. To confirm and extend the Southern analysis, clones from both pools were cultured until large ES cell colonies formed on the feeder cells. The relative amount of ES cell DNA was then much

FIG. 2 a, Southern analysis of PCR-amplified genomic DNA from microinjected $3 T 3$ cell pools (lanes $1-3$, for details see text) and plasmid pH1.1/O-BF (lane 4). $b$, Southern analysis of PCR-amplified genomic DNA from microinjected D3 cell pools. $c$, Schematic representation of plasmid $p \mathrm{H} 1.1 / 0-B F . A 3,643-\mathrm{bp}$ $5^{\prime}$ BamHI-Fspl 3' fragment of the Hox 1.1 gene (nucleotides $-1,711$ to $+1,932$ relative to the transiational start codon) was cloned into Bluescript (Stratagene) and the disrupting oligonucleotide was cloned into the EcoR site of the homoeobox.

METHODS. The construct outlined in Fig. 1 was microinjected into the nucleus ${ }^{13}$ of 3T3 or D3 cells (3T3: $200-600$ cells; D3: $50-200$ cells) at a concentration of $300 \mathrm{ng} \mathrm{ml}^{-1}$, corresponding to approximately five DNA molecules per cell with an injection volume of $10^{-11} \mathrm{ml}$. The cells were grown for 4-7 days after microinjection, treated with trypsin and replated using the same dish. The cells were collected again after 4-7 days. Half of these were used for DNA analysis and the rest stored in liquid nitrogen. Genomic DNA or control plasmid ( $1 \mu \mathrm{g}$ and $15 \mathrm{pg}$, respectively) was subjected to 30 PCR cycles using heat-stable Taq-polymerase ${ }^{14}$ in a $50 \mu$ reaction mixture containing $10 \%$ dimethyl sulphoxide, $67 \mathrm{mM}$ Tris- $\mathrm{HCl}\left(\mathrm{pH} 8.8\right.$ ), $16.6 \mathrm{mM} \mathrm{NH}_{4} \mathrm{SO}_{4}, 6.7 \mathrm{mM}$ $\mathrm{MgCl}_{2}, 10 \mathrm{mM}$ 2-mercaptoethanol, $170 \mu \mathrm{g} \mathrm{ml} \mathrm{H}^{-1}$ bovine serum albumin (BSA), $45 \mu \mathrm{M}$ each of the deoxyribonucleotide triphosphates (dATP, dCTP, dTTP, dGTP) and $0.5 \mu \mathrm{M}$ of each primer. Samples were overlaid with several drops of paraffin to prevent condensation. In each cycle, samples were heated to $91.5^{\circ} \mathrm{C}$ for $1 \mathrm{~min}$ to denature the DNA, cooled to $55^{\circ} \mathrm{C}$ for $1 \mathrm{~min}$ to anneal the primer and heated to $70^{\circ} \mathrm{C}$ for $6 \mathrm{~min}$ to activate the polymerase. Aliquots of the reaction mix $(10 \mu l)$ were electrophoresed in a $1 \%$ agarose gel, transferred to Gene Screen Plus (Dupont) and hybridized in $50 \%$ formamide, $1 \mathrm{M} \mathrm{NaCl}$ and $1 \% \mathrm{SDS}$. The hybridization probe was ${ }^{32} \mathrm{P}$-labelled to a specific higher as demonstrated in Fig. $3 b$ and $c$. A Southern blot of Kpn I digested DNA from a clonal line derived from pool 1 was hybridized to probe 1 (Fig. $3 b$, lane 1) and probe 2 (Fig. $3 b$, lane 2) (see Fig. $3 c$ for details of the probes). The relative intensities of the $5.4-\mathrm{kb}$ and the $8.6-\mathrm{kb}$ bands, corresponding to the $5^{\prime}$ and $3^{\prime}$ fragments, respectively (see Fig. $3 d$ ), compared with that of the 14-kb fragment, show that at least $50 \%$ of the ES cells carry the mutated allele. DNA of a clonal line from pool 7 was digested with StuI/KpnI (Fig. 3c, lane 1) and KpnI (Fig. 3c, lane 2) and Southern blots were subsequently hybrid-
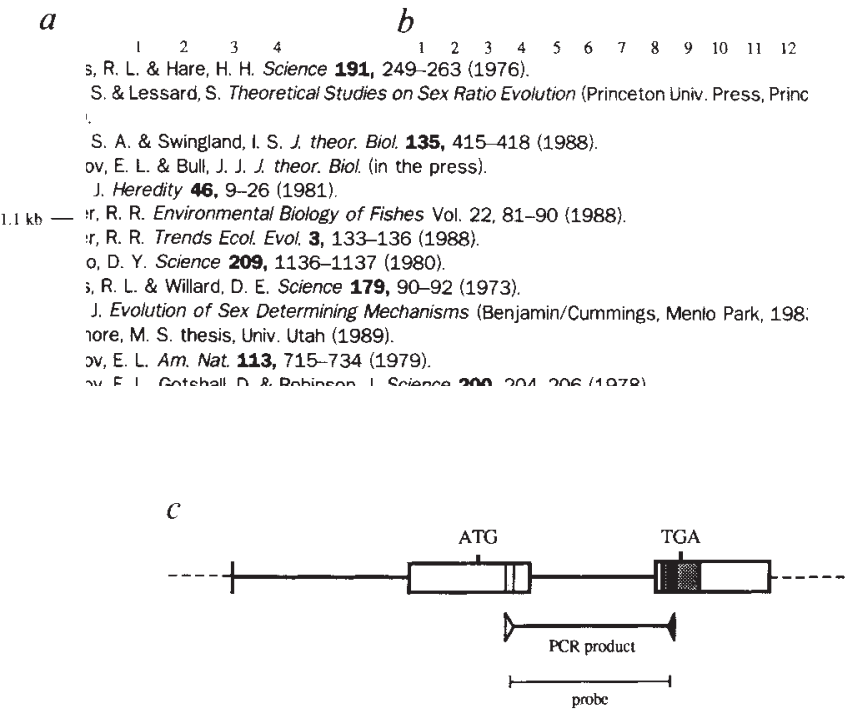

activity of $1 \times 10^{9}$ c.p.m. $\mu g^{-1}$ using a Multiprime kit (Amersham) and was used at $5 \times 10^{5}$ c.p.m. $\mathrm{ml}^{-1}$. Filters were washed in $2 \times$ SSC, then $2 \times$ SSC- $1 \%$ SDS and finally in $0.1 \times S S C$ at $65^{\circ} \mathrm{C}$. Exposure time, $2(a)$ and 5 hours (b).
FIG. 3 a, Southern analysis of Kpnl digests of genomic DNA from cloned ES cells hybridized with probe 1. Lane 1, clone showing the 5.4-kb band indicative of a homologous recombination event (for details see text). Lanes 2,3 and 4, clones in which no mutant Hox 1.1 allele can be detected. $b$, Southern analysis of an ES-cell clone from pool 1 . The clone was gown to a high density to minimize contamination by DNA from feeder cells. Southern blots of Kpnl-digested DNA. hybridized to probe 1 (lane 1 ) and 2 (lane 2), show the expected bands at $5.4-\mathrm{kb}$ and $8.6 \mathrm{~kb}$, respectively. $C$, Southern analysis of an ES cell clone from pool 7. Genomic DNA was digested with Stul/Kpnl (lane 1) or Konl (lane 2) and hybridized to probe 3 . d, Schematic representation of the mutant and normal Hox 1.1 allele and the expected restriction fragments, indicating the replacement of the EcoRl site by a Kpnl site in the second exon of the mutant allele. Abbreviations K, Kpnl; E, EcoRl; F, Fspl; S, Stul. METHODS. Single ES cells were picked with glass capillaries ${ }^{17}$ and plated into 96-well dishes. ES-cell colonies were subsequently expanded into $1.5-\mathrm{cm}, 24-$ well dishes and then into $6-\mathrm{cm}$ tissue-culture dishes. DNA was isolated from half of these cells and the other half was stored in liquid nitrogen. During the entire cloning procedure ES cells were cultured on feeder cells. Aliquots $(10 \mu \mathrm{g})$ of genomic DNA were digested with $\mathrm{Kpnl}$, electrophoresed in $0.8 \%$ agarose gels and transferred to Gene Screen Plus. Filters were hybridized to ${ }^{32}$ P.labelled probes and washed under the conditions described in Fig. 2

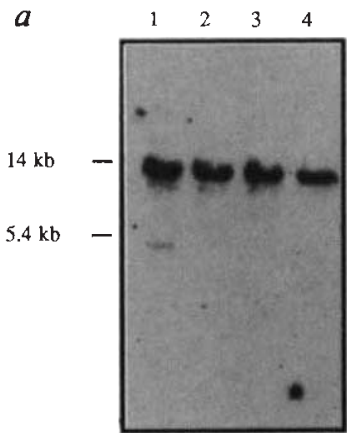

$b$

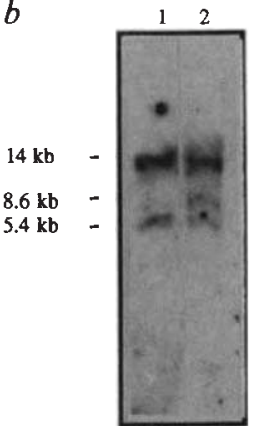

$d$

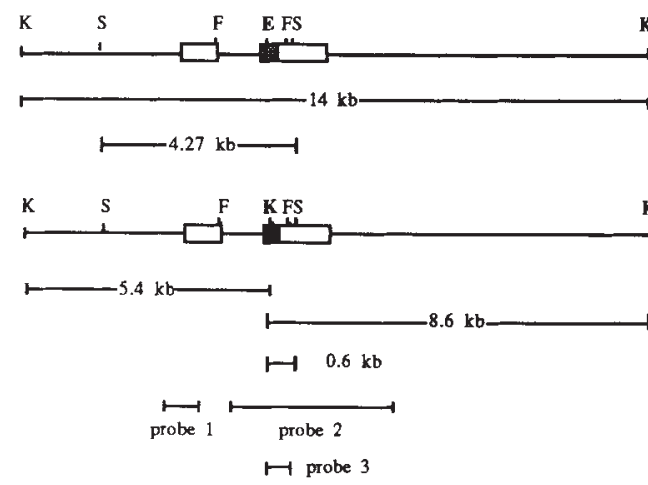

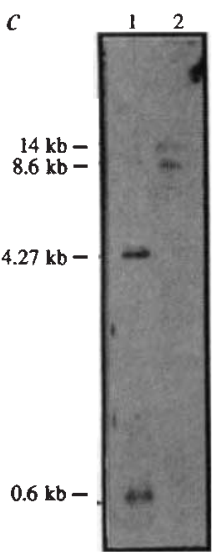

normal allele

mutated alleke 


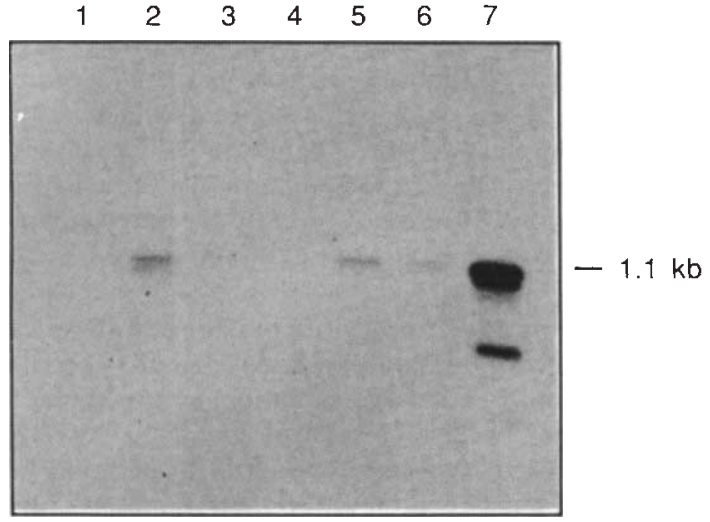

FIG. 4 Tail biopsis of two litters derived from blastocysts, which were microinjected with the clonal mutant ES cell line described in Fig. $3 a$ and $b$ (lanes 1-4, litter lanes 5-7, litter 2). $100 \mathrm{ng}$ of genomic DNA was subjected to 30 PCR cycles as described in Fig. 2. Lanes 2, 5,6 and 7 exhibit the amplification of the $1.1 \mathrm{~kb}$ fragment indicative of the mutant allele (see Figs 1 and 2). METHODS. Mice from two litters were separated after weaning and their tails were cut with new razor blades to prevent contamination. DNA was isolated by standard procedures and analysed by PCR as described in Fig. 2. Exposure time was 5 hours.

ized to probe 3 (see Fig. $3 d$ ). The resultant $600-\mathrm{bp}$ and $4.27-\mathrm{kb}$ fragments are derived from the mutated and normal alleles, respectively (see Fig. $3 d$ for details). The mutated and the normal allele are represented in equal amounts in the $S t u I / K p n I$ and the KpnI digest (Fig. $3 c$, lanes 1 and 2) indicating that the ES cells from pool 7 are clonal. A karyotype analysis of the clone derived from pool 1 revealed that approximately $70 \%$ of the cells contained 40 chromosomes (data not shown). Therefore we believe that the lower representation of the mutated allele in this line is due to contamination with non-mutated ES cells and does not reflect a chromosomal instability. We estimate that the ratio of homologous to illegitimate recombination is about $1: 30$, as about $20 \%$ of microinjected cells become stable transformants after nuclear microinjection ${ }^{13}$ and homologous recombination occurs in 1 per 150 microinjected cells. This is surprisingly high, relative to the results of comparable studies ${ }^{11,18,19}$ As the parameters that affect the frequency of homologous recombination are not well defined, the explanation for this difference must await further studies.

Our results probably reflect two main differences between our studies and earlier ones. First, the ratio of the heterologous to homologous sequences is very low in the construct used for microinjection. It has been demonstrated that the length of homology is directly proportional to the frequency of gene conversion $^{11}$, whereas the length of the heterology is inversely proportional $^{11,14}$. The other factor favouring high frequency could be the sensitivity of our analysis. We avoided potential problems associated with the requirement for marker gene expression by direct analysis of the DNA target with the highly sensitive PCR method which can potentially detect any gene conversion event. This should make in vivo mutagenesis an easy and efficient method for dissecting mammalian developmental pathways. Furthermore, it allows the introduction of more specific mutations, because conditions for the PCR analysis can be selected to detect even single base-pair exchanges ${ }^{10}$. These would be particularly desirable in cases where insertional inactivation of a gene leads to dominant lethal mutations. It should also be possible to generate mouse models for human diseases in which single amino acids are exchanged and to alter the expression pattern of a gene by modifying transcription-factor binding sites. Finally, the method is of potential interest for human somatic gene therapy, because it allows the repair of defective genes without the introduction of other foreign DNA sequences.
The cells containing the mutant allele showed no alterations in their morphology or in their growth properties (data not shown). The ES cells were microinjected into blastocysts of C57BL/6 mice and two litters with a total of four chimaeric animals were obtained. To confirm that the chimaeras contained the mutant allele, tail DNA was analysed by PCR (Fig. 4). In all four chimaeric animals, the expected 1.1-kb fragment was seen after amplification with primers 1 and 2 (Fig. 4, lanes 2, 5,6 and 7). At present all are healthy and show no abnormalities. These results show that homologous recombination of nonselectable genes can be obtained with high frequency in ES cells which can then be used to produce chimaeric animals. As relatively few cells are required for microinjection, PCR analysis and cloning, the number of individual cell manipulations is small. This reduces the possible accumulation of chromosomal aberrations and maintains a high frequency of germ line contribution. Thus, mouse mutants lacking an intact Hox 1.1 gene can be bred from chimaeric animals in order to determine gene function. By analogy, functional analysis through mutation of any gene may now be possible.

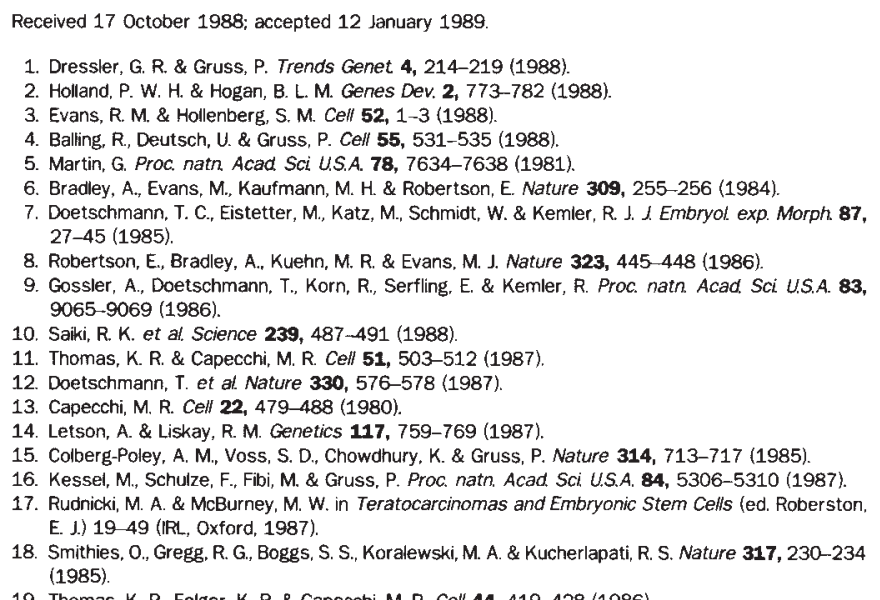

19. Thomas, K. R., Folger, K. R. \& Capecchi, M. R. Cell 44, 419428 (1986)

ACKNOWLEDGEMENTS. We thank Dr Rolf Kemler for the D3 cell line and Drs Colin Stewart, Lindsay Williams and Erwin Wagner for the introduction to blastocyst injection. We also thank Sabine Geisendorf for technical assistance, Dr Michael Kessel for helpful discussions and Drs Greg Dressler and Corrinne Lobe for reading the manuscript. This work was supported by the Max-Planck-Society and the Deutsche Forschungsgemeinschaft.

\section{Production of a mutation in mouse En-2 gene by homologous recombination in embryonic stem cells}

\author{
Alexandra L. Joyner, William C. Skarnes \\ 8. Janet Rossant
}

Division of Molecular and Development Biology,

Mount Sinai Hospital Research Institute, 600 University Avenue,

Toronto, Ontario, Canada M5G $1 \times 5$ and

Department of Medical Genetics, University of Toronto

A FULL understanding of the function of genes that control developmental events can be obtained only by a combination of molecular and mutational analysis. One putative developmental gene is the mouse engrailed-like gene $E n-2$, which was isolated by virtue of its extensive homology to Drosophila engrailed, which contributes to the control of segmentation in the developing insect ${ }^{1}$. Our hybridization analysis in situ has revealed that expression of $E n-2$ is restricted to a specific domain of the developing central nervous system from 8 days of development $\mathrm{on}^{2}$, indicating a role 